\title{
Increased risk of mycobacterial infections associated with anti-rheumatic medications
}

\author{
Sarah K Brode, ${ }^{1,2,3}$ Frances B Jamieson, ${ }^{4,5}$ Ryan $\mathrm{Ng}^{6}{ }^{6}$ Michael A Campitelli, ${ }^{6}$ \\ Jeffrey C Kwong, 4,6,7,8 J Michael Paterson, 6,9,10 Ping Li, ${ }^{6}$ Alex Marchand-Austin, ${ }^{4}$ \\ Claire Bombardier, ${ }^{9,11}$ Theodore K Marras ${ }^{1,3}$
}

\begin{abstract}
- Additional material is published online only. To view please visit the journal online (http://dx.doi.org/10.1136/ thoraxjnl-2014-206470).

For numbered affiliations see end of article.
\end{abstract}

\section{Correspondence to}

Dr Theodore K Marras, Toronto Western Hospital 7E 452, 399 Bathurst Street, Toronto, Ontario, Canada M5T 2S8; ted.marras@uhn.ca

Received 23 October 2014 Revised 25 February 2015 Accepted 31 March 2015 Published Online First 24 April 2015
CrossMark

To cite: Brode SK Jamieson $\mathrm{FB}, \mathrm{Ng} \mathrm{R}$, et al. Thorax 2015;70:677-682.

\section{ABSTRACT}

Rationale Anti-tumour necrosis factor (TNF) agents and other anti-rheumatic medications increase the risk of TB in rheumatoid arthritis (RA). Whether they increase the risk of infections with nontuberculous mycobacteria (NTM) is uncertain.

Objectives To determine the effect of anti-TNF therapy and other anti-rheumatic drugs on the risk of NTM disease and TB in older patients with RA.

Methods Population-based nested case-control study among Ontario seniors aged $\geq 67$ years with RA who were prescribed at least one anti-rheumatic medication between 2001 and 2011. We identified cases of TB and NTM disease microbiologically and identified drug exposures using linked prescription drug claims. We estimated ORs using conditional logistic regression, controlling for several potential confounders.

Measurements and main results Among 56269 older adults with RA, we identified 37 cases of TB and 211 cases of NTM disease; each case was matched to up to 10 controls. Individuals with TB or NTM disease were both more likely to be using anti-TNF therapy (compared with non-use); adjusted ORs (95\% Cls) were 5.04 (1.27 to 20.0) and 2.19 (1.10 to 4.37), respectively. Exposure to leflunomide and other antirheumatic drugs with high immunosuppressing potential also were associated with both TB and NTM disease, while oral corticosteroids and hydroxychloroquine were associated with NTM disease.

Conclusions Anti-TNF use is associated with increased risk of both TB and NTM disease, but appears to be a relatively greater risk for TB. Several other anti-rheumatic drugs were also associated with mycobacterial infections.

\section{INTRODUCTION}

Rheumatoid arthritis (RA) is a chronic autoimmune disorder affecting approximately $1 \%$ of all people. ${ }^{1}$ Over a decade ago, treatment for this disabling condition was revolutionised by the 'biological' agents, including anti-tumour necrosis factor (TNF) agents. This class of medications reduces the action of TNF- $\alpha$, a pro-inflammatory cytokine that causes synovitis in RA, but also plays a key role in the immune response to mycobacteria.

Numerous studies from around the world have demonstrated that anti-TNF therapy is associated with an increased risk of TB. ${ }^{2-4}$ However, patients with RA also are at higher risk for TB than the general population, ${ }^{5}$ perhaps due to both

\section{Key messages}

What is the key question?

- Do anti-tumour necrosis factor (TNF) and other anti-rheumatic drugs increase the risk of nontuberculous mycobacterial (NTM) disease and TB in older patients with rheumatoid arthritis (RA)?

What is the bottom line?

- Anti-TNF use is associated with increased risk of both TB and NTM disease; several other anti-rheumatic drugs are also associated with mycobacterial infections.

Why read on?

- This is the first study to describe an association between anti-TNF use and NTM disease after controlling for several potential confounders, and the first study to look at the relationship between other anti-rheumatic drugs and NTM disease.

anti-rheumatic medications ${ }^{4}$ and immune dysregulation intrinsic to the disease. ${ }^{6}$ The association between nontuberculous mycobacterial (NTM) disease, RA and immunosuppressive medications is less clear. NTM may act as opportunistic pathogens, exploiting airway mucosal ${ }^{7}$ or generalised immunodeficiency states, ${ }^{8}$ and so may pose a risk to patients with RA. Clinical confusion often arises around NTM disease as a mimic of TB; the former is more common in high-income countries and more difficult to treat. It is therefore important to determine if patients with RA using immunosuppressives are at increased risk of NTM, but few studies have examined this issue. ${ }^{9}$ In Ontario, Canada, persons with RA were found to have a twofold risk of NTM disease and TB compared with persons without RA, and experienced NTM disease far more commonly than TB. ${ }^{5}$ The main objective of this study was to quantify the risk of NTM isolation/disease and TB associated with anti-TNF use in patients with RA.

\section{METHODS}

Study population and setting

We performed a population-based nested casecontrol study using linked health administrative 
and laboratory data from the province of Ontario, Canada. Ontario residents entered the cohort on or after 1 January 2001, if they had RA, were $\geq 67$ years old, and filled at least one prescription for a traditional disease modifying anti-rheumatic drug (DMARD), a biological agent (a genetically engineered protein with a specific immune system target), or a non-steroidal anti-inflammatory drug (NSAID). Cohort patients were followed until the earliest of emigration, death or 31 December 2011. Ontario is Canada's most populous province (13.5 million in 2013), is ethnically diverse and has a single-payer healthcare system providing universal access to hospital care, physician services and prescription drugs to adults aged $\geq 65$ years. We included only individuals aged $\geq 67$ years to allow a 2 -year lookback for prescription drug use. Ethics approvals were from the Research Ethics Boards at the University of Toronto (26090), University Health Network (11-1018-AE) and Sunnybrook Health Sciences Centre, all in Toronto, Canada.

\section{Data sources}

We used the Canadian Institute for Health Information's Discharge Abstract Database for diagnostic information recorded during admissions to acute care hospitals and the Ontario Health Insurance Plan physician billing claims database for patient services to define RA and comorbidities. RA was identified using a validated diagnostic code algorithm, comprising $\geq 1$ hospitalisation with a diagnosis code for RA, or three physician claims with RA diagnosis codes in $\leq 2$ years $(\geq 1$ claim by a specialist). This algorithm has a sensitivity of $78 \%$ and a specificity of $100 \% .{ }^{10}$ Prescription drug exposures were identified using the Ontario Drug Benefit (ODB) database, which contains records of outpatient prescription medications dispensed to Ontarians aged $\geq 65$ years. Demographic information was obtained from the Ontario Registered Persons Database. Cases of mycobacterial infection were identified by linking individual patient records from the health administrative databases with the Public Health Ontario Laboratory database, which includes isolates from $100 \%$ of TB cases and approximately $95 \%$ of NTM cases in Ontario. ${ }^{11}$ Microbiological methods are described in online supplementary appendix 1.

\section{Identification of case and control patients}

Case patients were defined as follows: (a) TB, any culture positive for Mycobacterium tuberculosis complex organisms; (b) NTM isolation, any culture positive for NTM; and (c) NTM disease, NTM isolation from $\geq 2$ sputum or $\geq 1$ bronchoscopy or lung biopsy specimen (a validated microbiological surrogate for pulmonary NTM disease with a positive predictive value ranging between $69.5 \%$ and $90 \%$ in general populations ${ }^{12-15}$ and $100 \%$ in patients with RA on anti-TNF therapy ${ }^{16}$ ) or any positive culture from a normally sterile body site, including pleura. ${ }^{8}$ We excluded individuals with NTM isolated at any time before cohort entry $(n=317)$ or prior TB $(n=31)$ within the past $<18$ months. We also excluded all Mycobacterium gordonae isolations as likely contamination.

For cases, the index date was defined as the date the first positive mycobacterial culture was isolated. Each case was matched to up to 10 controls within the cohort of patients with RA on the index date according to age ( \pm 365 days), sex, year of cohort entry and duration of RA (within quartile: 0-3 years, 4-8 years, 9-13 years or $\geq 14$ years). Because of the large sample available to us, we chose to match up to 10 controls, to increase precision of results. When fewer than 10 controls could be matched to a case, we used only the controls that could be matched, without changing the matching algorithm. Cases could serve as controls before becoming a case, but controls were sampled without replacement. Controls were assigned the index date of their corresponding case patient.

\section{Drug exposure definitions}

The anti-TNF agents we studied were infliximab, adalimumab and etanercept. All non-biological anti-rheumatic drugs paid for by the ODB Program during the study period were also studied (oral corticosteroids, methotrexate, leflunomide, sulfasalazine, hydroxychloroquine, cyclophosphamide, azathioprine, ciclosporin, mycophenolate, chlorambucil, gold, penicillamine and NSAIDs). Because of a potential association between gastrooesophageal reflux disease (GORD) and pulmonary NTM infection, ${ }^{17}$ proton pump inhibitor (PPI) use was studied for NTM outcomes, but not TB. Individuals were considered exposed to an injectable medication starting on the prescription date and ending after five half-lives. Individuals were considered exposed to an oral medication (all medications other than anti-TNF agents) starting on the prescription date and ending after the number of days supplied on the prescription claim. For the primary analysis examining anti-TNF use, current use was defined as end of exposure $\leq 6$ months prior to index date, past use was defined as end of exposure 6-18 months prior to index date and no use was defined as never use or end of exposure $>18$ months prior to index date. 'Current use' extended 6 months beyond exposure to allow for delay after infection date to symptom onset, physician's request for specimen collection, collection of the specimen and specimen receipt in the laboratory. Others have defined 'current use' as 90 days beyond exposure, but found no significant difference between a 90-day and 6-month definition. ${ }^{9}$ We included a 'past use' exposure category because of the indolent nature of NTM infections, assuming that many patients experience a $>6$-month delay between infection and symptom onset/diagnosis. ${ }^{18}$ For the secondary analysis examining other anti-rheumatic medications, current use was defined as above, and non-current use was defined as non-use or end of exposure $>6$ months prior to index date. Corticosteroid dose was computed based upon the most recently filled prescription and converted to average daily prednisone equivalents: (1) low dose, $\leq 9 \mathrm{mg} / \mathrm{day}$; (2) medium dose, 10-19 mg/day and (3) high dose, $\geq 20 \mathrm{mg} /$ day.

\section{Statistical analysis}

We used conditional logistic regression to estimate the crude and adjusted ORs and 95\% CIs to quantify associations between TB, NTM isolation, NTM disease and exposure to specific anti-rheumatic drugs. The primary analysis compared current and past users versus non-users of anti-TNF agents. The preplanned secondary analysis compared current versus noncurrent users of other anti-rheumatic drugs. The multivariable model was adjusted for covariates possibly associated with $\mathrm{TB}$ or NTM, including income, rurality of patient residence, presence of extra-articular features of RA (grouped into lung, skin and other), ${ }^{19}$ other anti-rheumatic medication use, aggregated diagnostic groups (ADGs) (a general measure of comorbidity) ${ }^{20}$ and comorbidities. ADGs were determined from inpatient and outpatient health records from 2 years prior to the index date, using the Johns Hopkins Adjusted Clinical Group Case-Mix System. Comorbidities included diabetes and chronic kidney disease for TB, and asthma, COPD and GORD for NTM. Comorbidities were identified using validated algorithms, ${ }^{21-25}$ looking back from cohort entry to 1 April 1991 (the earliest date available). If definitions were not met, comorbidities were assumed to be absent. Because data capture was complete for 
our variables, no allowance for handling of missing data was required. Analyses were performed using SAS V.9.3 (SAS Institute, Cary, North Carolina, USA). All tests were two-tailed with a type 1 error $(\alpha)$ rate of $5 \%$.

\section{RESULTS}

We identified 61827 elderly adults with RA during the study period (figure 1). We excluded 5558 because they did not fill at least one prescription for a DMARD, biological or NSAID, leaving 56269 individuals. Within this group, we identified 37 cases of TB who we matched to 363 controls, and 211 cases of NTM disease who we matched to 2071 controls. Findings regarding NTM isolation are presented in online supplementary appendix 2 , and NTM species causing disease are presented in online supplementary appendix 3 . TB cases were more likely to live in urban settings and have pulmonary extra-articular RA, overall comorbidities (higher ADGs), chronic kidney disease and diabetes than controls (table 1). ${ }^{26}$ NTM disease cases were more likely to live in urban settings, have pulmonary extra-articular RA, overall comorbidities, asthma, COPD and GORD than controls.

\section{Anti-TNF use}

Current anti-TNF users had significantly increased risk of TB compared with non-users (crude $\mathrm{OR}=6.44$ (2.02 to 20.6), adjusted $\mathrm{OR}=5.04$ (1.27 to 20.0)) (table 2). Current anti-TNF users also had significantly increased risk of NTM disease compared with non-users (crude $\mathrm{OR}=2.42$ (1.34 to 4.37), adjusted $\mathrm{OR}=2.19$ (1.10 to 4.37$)$ ), but the risk magnitude was numerically less than for TB. There were no anti-TNF past users among TB cases. Past anti-TNF use was not associated with NTM disease. Regarding site of infection, $89 \%$ of NTM disease was pulmonary, without significant difference between anti-TNF users (87\%) and non-users (89\%). Eighty-one per cent of TB was pulmonary, without significant difference between anti-TNF users $(83 \%)$ and non-users $(81 \%)$.

\section{Other anti-rheumatic medication use}

Several non-biological anti-rheumatic medications were infrequently used among both TB and NTM disease cases, and so were grouped into classes with high or low immunosuppressing potential based on clinical judgement (table 2).

Many anti-rheumatic medications were associated with an increased risk of TB and NTM disease in unadjusted analyses.

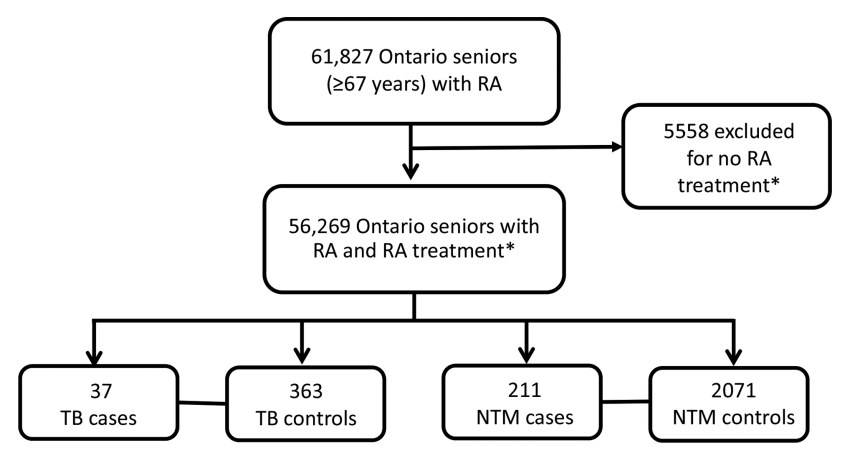

Figure 1 Study population. TB cases defined by any culture positive for Mycobacterium tuberculosis complex organisms; NTM disease cases defined by $\geq 2$ sputum, or $\geq 1$ bronchoscopy or lung biopsy specimen, or any positive culture from a normally sterile body site, including pleura. DMARD, disease modifying anti-rheumatic drug; NSAID, non-steroidal anti-inflammatory drug; NTM, nontuberculous mycobacterial; RA, rheumatoid arthritis. *RA treatment includes at least one filled prescription for a DMARD, biological or NSAID.
After full adjustment, only leflunomide (OR=4.02 (1.08 to $15.0)$ ) and drugs classed as highly immunosuppressive $(\mathrm{OR}=23.0(2.88$ to 184$))$ were associated with an increased risk of TB (table 2). High-dose oral corticosteroids ( $\mathrm{OR}=1.60(1.02$ to 2.52$)$ ), leflunomide ( $\mathrm{OR}=2.74$ (1.59 to 4.70$)$ ), hydroxychloroquine $(\mathrm{OR}=1.62$ (1.13 to 2.33$))$ and drugs classed as highly immunosuppressive $(\mathrm{OR}=2.92$ (1.26 to 6.79$)$ ) were associated with an increased risk of NTM disease after adjustment (table 2).

\section{DISCUSSION}

In our cohort of 56269 older adults with RA, current users of anti-TNF agents, were at increased risk of both TB and NTM disease. Because of higher rates of NTM disease in the RA cohort overall, there were more cases of NTM disease than TB in anti-TNF users, but the magnitude of the risk was larger for TB than for NTM disease (adjusted OR: 5.04 vs 2.19). Several other anti-rheumatic medications also increased the risk of $\mathrm{TB}$ and NTM disease to a similar magnitude as anti-TNF agents.

Several studies have reported an increased risk of $\mathrm{TB}$ in anti-TNF users; ${ }^{2-4}$ however, few examined the association between anti-TNF agents and NTM disease. The most rigorous of the latter group was a cohort study among members of one health maintenance organization (HMO) in California. ${ }^{9}$ In that study, incidence rates of TB and NTM in anti-TNF users were calculated, as were rates in the general HMO population and the unexposed RA population. Rates of both NTM disease and TB were higher in anti-TNF users than unexposed patients with RA, and NTM was more common than TB in anti-TNF users. However, this study did not adjust for potential confounders when comparing risks between exposed and unexposed patients. In addition, members of an HMO may not be representative of the general population. The findings of our population-based study expand upon their results by confirming that the risks of mycobacterial infections are increased in anti-TNF users compared with non-users, even after adjusting for several potential confounders.

Another interesting finding in our study was the increased risks of both TB and NTM disease associated with several other anti-rheumatic drugs. Previous work has shown an increased risk of TB with oral corticosteroids, methotrexate, leflunomide and ciclosporin. ${ }^{27}$ We also found an increased risk of TB with leflunomide and drugs with high immunosuppressing potential (including ciclosporin). However, after full adjustment, there was no association between oral corticosteroid or methotrexate use and TB in our study, perhaps due to the rarity of TB in our cohort.

Regarding NTM disease, there has been limited research on associations with non-biological anti-rheumatic drugs. One case-control study showed an increased risk of NTM associated with use of oral corticosteroids and immunomodulatory drugs, defined as biological and non-biological DMARDs. ${ }^{28}$ Case series have also suggested an increased risk of NTM in stem cell and solid organ transplantation recipients ${ }^{29}$ likely at least partially due to immunosuppressant use. However, no prior study has examined the risk of NTM disease associated with use of different anti-rheumatic drugs. Our findings suggest that several antirheumatic drugs increase the risk of NTM disease. Somewhat surprisingly, the risk of NTM associated with leflunomide and drugs classed as highly immunosuppressive was actually of larger magnitude than the risk with anti-TNF agents, although CIs overlapped.

The difference in anti-TNF-associated risk estimates between TB and NTM disease is intriguing. The larger OR for TB could be explained by imprecise risk estimates in the TB group due to the small number of $\mathrm{TB}$ cases, or alternatively, to differences in 
Table 1 Baseline characteristics on the index date for cases and controls*

\begin{tabular}{|c|c|c|c|c|c|c|c|c|}
\hline \multirow[b]{2}{*}{ Characteristic } & \multicolumn{4}{|l|}{ TB } & \multicolumn{4}{|c|}{ NTM disease } \\
\hline & $\begin{array}{l}\text { Cases } \\
\mathrm{N}=37\end{array}$ & $\begin{array}{l}\text { Controls } \\
\mathrm{N}=363\end{array}$ & SDMt & $p$ Value & $\begin{array}{l}\text { Cases } \\
\mathrm{N}=211\end{array}$ & $\begin{array}{l}\text { Controls } \\
\mathrm{N}=2071\end{array}$ & SDMt & p Value \\
\hline Female & $22(59)$ & $220(61)$ & 0.02 & 0.89 & $147(70)$ & $1452(70)$ & 0.01 & 0.89 \\
\hline Age, mean $\pm S D$ & $76.5 \pm 7.5$ & $76.2 \pm 7.1$ & 0.05 & 0.79 & $76.3 \pm 6.0$ & $76.1 \pm 5.8$ & 0.03 & 0.67 \\
\hline RA duration (years), mean \pm SD & $8.1 \pm 5.6$ & $8.5 \pm 5.5$ & 0.09 & 0.62 & $8.9 \pm 5.3$ & $8.9 \pm 5.2$ & 0.00 & 1.00 \\
\hline Income above median & $20(54)$ & $206(57)$ & 0.05 & 0.75 & $128(61)$ & $1193(58)$ & 0.06 & 0.39 \\
\hline Rural residency & $0(0)$ & $53(15)$ & 0.43 & 0.01 & $13(6)$ & $307(15)$ & 0.25 & $<0.001$ \\
\hline \multicolumn{9}{|l|}{ Extra-articular RA: } \\
\hline Lung & $6(16)$ & $25(7)$ & 0.35 & 0.04 & $44(21)$ & $143(7)$ & 0.51 & $<0.001$ \\
\hline Skin & $\leq 5(\leq 14)$ & $37(10)$ & 0.02 & 0.91 & $28(13)$ & $202(10)$ & 0.12 & 0.11 \\
\hline Other & $\leq 5(\leq 14)$ & $6(2)$ & 0.27 & 0.12 & $\leq 5(\leq 3)$ & $32(2)$ & 0.03 & 0.70 \\
\hline $\mathrm{ADGs}$, mean $\pm \mathrm{SD}$ & $11.8 \pm 3.5$ & $8.9 \pm 3.5$ & 0.81 & $<0.001$ & $11.2 \pm 3.2$ & $8.8 \pm 3.6$ & 0.67 & $<0.001$ \\
\hline Asthma & $57(16)$ & $6(16)$ & 0.01 & 0.93 & $89(42)$ & $374(18)$ & 0.61 & $<0.001$ \\
\hline COPD & $11(30)$ & 99 (27) & 0.05 & 0.75 & $135(64)$ & $616(30)$ & 0.75 & $<0.001$ \\
\hline GORD & $11(30)$ & $92(25)$ & 0.10 & 0.56 & $61(29)$ & $493(24)$ & 0.12 & 0.10 \\
\hline CKD & $6(16)$ & $37(10)$ & 0.18 & 0.29 & $26(12)$ & $211(10)$ & 0.07 & 0.33 \\
\hline Diabetes & $9(24)$ & 70 (19) & 0.13 & 0.46 & $49(23)$ & $509(25)$ & 0.03 & 0.66 \\
\hline
\end{tabular}

*Values are the number of patients (\%) unless otherwise indicated.

TSDM, standardised difference of the mean (mean difference as a percentage of the average SD) wherein $>0.1(10 \%)$ implies a meaningful imbalance between groups. ${ }^{26}$

ADGs, aggregated diagnostic groups; CKD, chronic kidney disease, GORD, gastro-oesophageal reflux disease; NTM, nontuberculous mycobacterial; RA, rheumatoid arthritis; SDM,

standardised difference of the mean.

pathophysiology between TB and NTM disease, such as the existence of latent infection. Anti-TNF therapy, by reducing macrophage ability to control growth of intracellular M. tuberculosis bacilli, ${ }^{30}$ increases the risk of progression of both latent and newly acquired TB infection. Through effects on latent TB, anti-TNF therapy facilitates emergence of disease reflecting a lifetime of prior exposure, an effect particularly relevant in our cohort of older people in a low-prevalence setting where latent TB infection (LTBI) progression largely drives TB rates. Regarding NTM, for which latent infection is not recognised, deleterious effects of anti-TNF therapy are likely limited to promoting progression of existing infection or acquisition of new infection. In our cohort, we removed all people with previously isolated NTM, which likely reduced the potential effect on disease progression. This may have limited our study to primarily examining drug effects on new infection, which may take many years to be fully expressed. Our speculation regarding LTBI presupposes imperfect screening for LTBI before anti-TNF therapy, a notion supported by reports of substantial discordance between tuberculin skin testing (TST) and interferon gamma release assay (IGRA), even among people without a potentially confounding history of BCG vaccination. ${ }^{31}$ Major discordance between tests suggests that testing for LTBI with a single test (either TST or IGRA), likely the most common practice in Ontario, fails to detect a substantial proportion of LTBI.

The rationale behind screening for and treating LTBI prior to anti-TNF therapy is clear and reduces the risk of TB disease. ${ }^{32}$ Screening patients with RA for NTM infection prior to anti-TNF and other high-risk anti-rheumatic drugs may also provide benefit. From the RA perspective, patients have increased rates of NTM, and immunosuppression is a risk factor for NTM disease, which may promote progression of preexisting unrecognised infection. From the NTM disease perspective, this infection significantly impairs quality of life, ${ }^{33}$ and its treatment is challenging, with low cure rates and high rates of drug toxicity. ${ }^{8}$ Considering the possibility of NTM infection in patients with RA before initiating high-risk anti-rheumatic drugs might identify pulmonary NTM infections that require antimicrobial therapy, closer monitoring and/or avoidance of high-risk immunosuppressive drugs. NTM screening in this setting may comprise a pulmonary and systemic symptom review and chest radiograph, both likely performed before initiating immunosuppression. Further testing might be considered based on the results of history and radiography. Current evidence may not yet be sufficient to recommend universal screening, but we think that this issue should be explored.

This study has several limitations. An important limitation is the absence of individual-level data on rheumatic disease activity, preventing separation of the effect of disease activity from anti-rheumatic drugs. Rheumatic disease activity, indicated by extra-articular RA and NSAID/cyclooxygenase (COX)-inhibitor use, increases the risk of infections. ${ }^{19}$ Although we adjusted for these factors, this proxy is of uncertain adequacy. ${ }^{19}$ It is therefore possible that the observed associations between antirheumatic drugs and mycobacterial infections are more related to rheumatic disease activity than they are to medication use. This might explain the observed association between NTM disease and hydroxychloroquine (OR: 1.62 (1.13 to 2.33)), a drug not typically associated with infection, and the lack of association with methotrexate (OR: 1.31 (0.93 to 1.86)), a drug known to increase risk of infections. ${ }^{34}$ Most Ontarians with RA who are on DMARDs other than methotrexate likely have more severe disease activity than those who only receive methotrexate because the Canadian Rheumatology Association recommends methotrexate as the first-line DMARD for the treatment of RA. $^{35}$

Another study limitation is the reliance on health administrative data to identify drug exposure. Outpatient medication adherence cannot be confirmed, and inpatient medication use is unavailable, so medication exposures could be over- or underestimated. Also, we defined TB and NTM disease based on microbiological data. Microbiological-based outcome definitions have excellent positive predictive value for TB $(100 \%),{ }^{16}$ but by ignoring clinically diagnosed culture-negative cases, their sensitivity is reduced (55\%-79\%). ${ }^{16}$ Regarding NTM disease, our microbiological-based outcome definition has shown good 
Table 2 ORs for TB and NTM disease according to anti-rheumatic medication use

\begin{tabular}{|c|c|c|c|c|c|c|c|c|c|c|c|c|}
\hline \multirow[b]{2}{*}{ Exposure } & \multicolumn{6}{|l|}{ TB } & \multicolumn{6}{|c|}{ NTM disease } \\
\hline & $\begin{array}{l}\text { TB cases } \\
\mathrm{N}=37\end{array}$ & $\begin{array}{l}\text { TB controls } \\
\mathrm{N}=363\end{array}$ & Crude OR $(95 \% \mathrm{Cl})$ & $p$ Value & Adjusted* OR (95\% Cl) & $p$ Value & $\begin{array}{l}\text { NTM cases } \\
\mathrm{N}=211\end{array}$ & $\begin{array}{l}\text { NTM controls } \\
\mathrm{N}=2071\end{array}$ & Crude OR (95\% CI) & $\mathrm{p}$ Value & Adjusted* OR (95\% Cl) & $\mathrm{p}$ Value \\
\hline \multicolumn{13}{|l|}{ Anti-TNF use } \\
\hline No use & $31(84)$ & $350(96)$ & 1.0 (ref) & & 1.0 (ref) & & $194(92)$ & 1997 (96) & 1.0 (ref) & & 1.0 (ref) & \\
\hline Past use & $0(0)$ & $0(0)$ & $\mathrm{N} / \mathrm{A}$ & & $\mathrm{N} / \mathrm{A}$ & & NR & $7(0.3)$ & 2.94 (0.61 to 14.2$)$ & 0.18 & 1.09 (0.15 to 8.09$)$ & 0.93 \\
\hline Current use & $6(16)$ & $13(4)$ & 6.44 (2.02 to 20.6$)$ & 0.002 & $5.04(1.27$ to 20.0$)$ & 0.02 & NR & $67(3)$ & $2.42(1.34$ to 4.37$)$ & 0.003 & 2.19 (1.10 to 4.37$)$ & 0.03 \\
\hline \multicolumn{13}{|l|}{ Oral corticosteroids } \\
\hline Non-current use & $15(40)$ & $242(66)$ & 1.0 (ref) & & 1.0 (ref) & & $106(50)$ & $1401(68)$ & 1.0 (ref) & & 1.0 (ref) & \\
\hline Low dose & $9(24)$ & $57(16)$ & 2.45 (1.04 to 5.81$)$ & 0.04 & 1.63 (0.60 to 4.44$)$ & 0.34 & $32(15)$ & $344(17)$ & $1.28(0.84$ to 1.93$)$ & 0.25 & 0.72 (0.45 to 1.14$)$ & 0.16 \\
\hline Mod dose & $6(16)$ & $23(6)$ & $3.80(1.33$ to 10.8$)$ & 0.01 & $2.53(0.55$ to 11.6$)$ & 0.23 & $31(15)$ & $142(7)$ & $2.97(1.90$ to 4.63$)$ & $<0.001$ & 1.66 (0.99 to 2.78$)$ & 0.05 \\
\hline High dose & 7 (19) & $41(11)$ & 2.72 (1.04 to 7.09$)$ & 0.04 & 2.73 (0.81 to 9.20$)$ & 0.11 & $42(20)$ & $184(9)$ & 3.24 (2.18 to 4.84$)$ & $<0.001$ & $1.60(1.02$ to 2.52$)$ & 0.04 \\
\hline \multicolumn{13}{|l|}{ Methotrexate } \\
\hline Non-current use & $25(68)$ & $256(70)$ & 1.0 (ref) & & 1.0 (ref) & & $136(64)$ & $1431(69)$ & 1.0 (ref) & & 1.0 (ref) & \\
\hline Current use & $12(32)$ & $107(30)$ & 1.19 (0.57 to 2.48$)$ & 0.65 & 1.10 (0.44 to 2.72 ) & 0.84 & $75(36)$ & $640(31)$ & $1.27(0.94$ to 1.71$)$ & 0.12 & 1.31 (0.93 to 1.86$)$ & 0.12 \\
\hline \multicolumn{13}{|l|}{ Leflunomide } \\
\hline Non-current use & $31(84)$ & $343(94)$ & 1.0 (ref) & & 1.0 (ref) & & $185(88)$ & 1977 (96) & 1.0 (ref) & & 1.0 (ref) & \\
\hline Current use & $6(16)$ & $20(6)$ & $3.50(1.28$ to 9.60$)$ & 0.01 & $4.02(1.08$ to 15.0$)$ & 0.04 & $26(12)$ & $94(4)$ & 3.05 (1.92 to 4.85$)$ & $<0.001$ & 2.74 (1.59 to 4.70$)$ & $<0.001$ \\
\hline \multicolumn{13}{|l|}{ Sulfasalazine } \\
\hline Non-current use & NR & $343(94)$ & 1.0 (ref) & & 1.0 (ref) & & 199 (94) & 1952 (94) & 1.0 (ref) & & 1.0 (ref) & \\
\hline Current use & NR & $20(6)$ & $1.43(0.40$ to 5.19$)$ & 0.58 & 1.98 (0.40 to 9.78$)$ & 0.40 & $12(6)$ & $119(6)$ & $1.01(0.55$ to 1.87$)$ & 0.98 & 0.89 (0.44 to 1.77$)$ & 0.73 \\
\hline \multicolumn{13}{|l|}{ Hydroxychloroquine } \\
\hline Non-current use & NR & $272(75)$ & 1.0 (ref) & & 1.0 (ref) & & $147(70)$ & $1606(78)$ & 1.0 (ref) & & 1.0 (ref) & \\
\hline Current use & NR & $91(25)$ & $0.36(0.13$ to 1.05$)$ & 0.06 & $0.17(0.04$ to 0.65$)$ & 0.01 & $64(30)$ & $465(22)$ & 1.55 (1.13 to 2.13$)$ & 0.007 & 1.62 (1.13 to 2.33$)$ & 0.009 \\
\hline \multicolumn{13}{|l|}{ High-risk DMARD† } \\
\hline Non-current use & NR & $356(98)$ & 1.0 (ref) & & 1.0 (ref) & & 199 (94) & 2034 (98) & 1.0 (ref) & & 1.0 (ref) & \\
\hline Current use & NR & $7(2)$ & 5.84 (1.46 to 23.4$)$ & 0.01 & $23.0(2.88$ to 184$)$ & 0.003 & $12(6)$ & $37(2)$ & 3.25 (1.62 to 6.52$)$ & $<0.001$ & $2.92(1.26$ to 6.79$)$ & 0.01 \\
\hline \multicolumn{13}{|l|}{ Low-risk DMARD $\ddagger$} \\
\hline Non-current use & NR & $356(98)$ & 1.0 (ref) & & 1.0 (ref) & & NR & 2039 (98) & 1.0 (ref) & & 1.0 (ref) & \\
\hline Current use & NR & $7(2)$ & $1.48(0.16$ to 13.6$)$ & 0.73 & 3.43 (0.26 to 45.7$)$ & 0.35 & NR & $32(2)$ & $1.58(0.61$ to 4.12$)$ & 0.35 & 1.94 (0.65 to 5.80$)$ & 0.24 \\
\hline \multicolumn{13}{|l|}{ NSAID } \\
\hline Non-current use & $26(70)$ & $219(60)$ & 1.0 (ref) & & 1.0 (ref) & & $129(61)$ & $1202(58)$ & 1.0 (ref) & & 1.0 (ref) & \\
\hline Current use & $11(30)$ & $144(40)$ & 0.62 (0.28 to 1.35$)$ & 0.23 & 0.79 (0.30 to 2.06$)$ & 0.62 & $82(39)$ & $869(42)$ & $0.90(0.67$ to 1.21$)$ & 0.47 & 0.94 (0.68 to 1.30$)$ & 0.71 \\
\hline \multicolumn{13}{|l|}{ PPI } \\
\hline Non-current use & NA & NA & NA & & NA & & $110(52)$ & $1210(58)$ & 1.0 (ref) & & 1.0 (ref) & \\
\hline Current use & NA & NA & NA & NA & NA & NA & $101(48)$ & $861(42)$ & 1.29 (0.97 to 1.72$)$ & 0.08 & 0.77 (0.55 to 1.08$)$ & 0.12 \\
\hline
\end{tabular}

Bolded OR values are statistically significantly different than reference group. gastro-oesophageal reflux disease).

HHigh-risk DMARDs are a grouping of drugs with high immunosuppressing potential, including cyclophosphamide, azathioprine, ciclosporin, mycophenolate and chlorambucil.

¥Low-risk DMARDs have low immunosuppressing potential and include gold and penicillamine.

anti-TNF, anti-tumour necrosis factor; DMARD, disease modifying anti-rheumatic drug; NA, not assessed; NR, not reportable (in accordance with data use restrictions, cells containing fewer than six individuals cannot be reported); NSAID, non-steroidal anti-inflammatory; NTM, nontuberculous mycobacterial; PPI, proton pump inhibitor; ref, reference group. 
positive predictive value $(69.5 \%-100 \%),{ }^{12-16}$ but still might have misclassified some patients with NTM colonisation as having disease, reducing the validity of risk estimates.

In summary, although it is difficult to distinguish risks conferred by anti-rheumatic drugs from disease activity among Ontario seniors with RA, current anti-TNF use is associated with an increased risk of both TB and NTM. Leflunomide and drugs with high immunosuppressing potential are risk factors for both TB and NTM, while high-dose corticosteroids and hydroxychloroquine are risk factors for NTM. Potential approaches and benefits of screening for NTM disease in this population should be investigated.

\section{Author affiliations}

${ }^{1}$ Joint Division of Respirology, Department of Medicine, University Health Network and Mount Sinai Hospital, Toronto, Ontario, Canada

${ }^{2}$ West Park Healthcare Centre, Toronto, Ontario, Canada

${ }^{3}$ Department of Medicine, University of Toronto, Toronto, Ontario, Canada

${ }^{4}$ Public Health Ontario, Toronto, Ontario, Canada

${ }^{5}$ Department of Laboratory Medicine and Pathobiology, University of Toronto,

Toronto, Ontario, Canada

${ }^{6}$ Institute for Clinical Evaluative Sciences, Toronto, Ontario, Canada

${ }^{7}$ Department of Family and Community Medicine, University of Toronto, Toronto,

Ontario, Canada

${ }^{8}$ Toronto Western Family Health Team, University Health Network, Toronto, Ontario, Canada

${ }^{9}$ Institute of Health Policy, Management and Evaluation, University of Toronto, Toronto, Ontario, Canada

${ }^{10}$ Department of Family Medicine, McMaster University, Hamilton, Ontario, Canada

${ }^{11}$ Toronto General Hospital Research Institute, Toronto, Ontario, Canada

Acknowledgements We would like to thank the Data Quality and Information Management department at the Institute for Clinical Evaluative Sciences (ICES) for linking the mycobacteria testing data from the Public Health Ontario (PHO) Laboratories to the provincial health administrative data.

Contributors All authors contributed substantially to the conception and design of this work. AM-A and RN performed the data acquisition. RN, MAC and PL performed the analysis. SKB and TKM drafted the article. All authors revised the article critically for important intellectual content and gave final approval of the version to be published. The sponsors had no role in the development of the research or the manuscript.

Funding The authors acknowledge and thank the Ontario Thoracic Society and the Ontario Lung Association for funding support provided for this research study. This research was also supported by the Institute for Clinical Evaluative Sciences (ICES), a non-profit research institute funded by the Ontario Ministry of Health and Long-Term Care. The opinions, results and conclusions reported in this paper are those of the authors and are independent from the funding sources. No endorsement by ICES or the Ontario Ministry of Health and Long-Term Care is intended or should be inferred.

Competing interests None declared.

Ethics approval Research Ethics Boards at University of Toronto (26090), University Health Network (11-1018-AE) and Sunnybrook Health Sciences Centre.

Provenance and peer review Not commissioned; externally peer reviewed.

\section{REFERENCES}

1 Spector TD. Rheumatoid arthritis. Rheum Dis Clin North Am 1990;16:513-37.

2 Dixon WG, Hyrich KL, Watson KD, et al. Drug-specific risk of tuberculosis in patients with rheumatoid arthritis treated with anti-TNF therapy: results from the British Society for Rheumatology Biologics Register (BSRBR). Ann Rheum Dis 2010;69:522-8.

3 Tubach F, Salmon D, Ravaud P, et al. Risk of tuberculosis is higher with anti-tumor necrosis factor monoclonal antibody therapy than with soluble tumor necrosis factor receptor therapy: the three-year prospective French Research Axed on Tolerance of Biotherapies registry. Arthritis Rheum 2009;60:1884-94.

4 Brassard P, Kezouh A, Suissa S. Antirheumatic drugs and the risk of tuberculosis. Clin Infect Dis 2006;43:717-22.

5 Brode SK, Jamieson FB, $\mathrm{Ng} \mathrm{R}$, et al. Risk of mycobacterial infections associated with rheumatoid arthritis in Ontario, Canada. Chest 2014;146:563-72.

6 Wagner UG, Koetz K, Weyand CM, et al. Perturbation of the T cell repertoire in rheumatoid arthritis. Proc Natl Acad Sci USA 1998;95:14447-52.
7 Fowler CJ, Olivier KN, Leung JM, et al. Abnormal nasal nitric oxide production, ciliary beat frequency, and Toll-like receptor response in pulmonary nontuberculous mycobacterial disease epithelium. Am J Respir Crit Care Med 2013;187:1374-81.

8 Griffith DE, Aksamit T, Brown-Elliott BA, et al. An official ATS/IDSA statement: diagnosis, treatment, and prevention of nontuberculous mycobacterial diseases. Am J Respir Crit Care Med 2007:175:367-416.

9 Winthrop K, Baxter R, Liu L, et al. Mycobacterial diseases and antitumour necrosis factor therapy in USA. Ann Rheum Dis 2013:72:37-42.

10 Widdifield J, Bombardier C, Bernatsky S, et al. An administrative data validation study of the accuracy of algorithms for identifying rheumatoid arthritis: the influence of the reference standard on algorithm performance. BMC Musculoskelet Disord 2014;15:216.

11 AlHouqani $M$, Jamieson $F$, Cheodore $P$, et al. Isolation prevalence of pulmonary nontuberculous mycobacteria in Ontario 2007. Can Respir J 2011;18:19-24.

12 Andrejak C, Thomsen VO, Johansen IS, et al. Nontuberculous pulmonary mycobacteriosis in Denmark: incidence and prognostic factors. Am J Respir Crit Care Med 2010;181:514-21.

13 Prevots DR, Shaw PA, Strickland D, et al. Nontuberculous mycobacterial lung disease prevalence at four integrated health care delivery systems. Am J Respir Crit Care Med 2010;182:970-6.

14 Winthrop KL, McNelley E, Kendall B, et al. Pulmonary nontuberculous mycobacterial disease prevalence and clinical features: an emerging public health disease. Am J Respir Crit Care Med 2010;182:977-82.

15 Marras TK, Mehta M, Chedore $P$, et al. Nontuberculous mycobacterial lung infections in Ontario, Canada: clinical and microbiological characteristics. Lung 2010;188:289-99.

16 Winthrop KL, Baxter R, Liu L, et al. The reliability of diagnostic coding and laboratory data to identify tuberculosis and nontuberculous mycobacterial disease among rheumatoid arthritis patients using anti-tumor necrosis factor therapy. Pharmacoepidemiol Drug Saf 2011;20:229-35.

17 Thomson RM, Armstrong JG, Looke DF. Gastroesophageal reflux disease, acid suppression, and Mycobacterium avium complex pulmonary disease. Chest 2007;131:1166-72.

18 Kim RD, Greenberg DE, Ehrmantraut ME, et al. Pulmonary nontuberculous mycobacterial disease: prospective study of a distinct preexisting syndrome. Am J Respir Crit Care Med 2008;178:1066-74.

19 Widdifield J, Bernatsky S, Paterson JM, et al. Serious infections in a population-based cohort of 86,039 seniors with rheumatoid arthritis. Arthritis Care Res (Hoboken) 2013;65:353-61.

20 Reid RJ, MacWilliam L, Verhulst L, et al. Performance of the ACG case-mix system in two Canadian provinces. Med Care 2001;39:86-99.

21 Hux JE, Ivis F, Flintoft $V$, et al. Diabetes in Ontario: determination of prevalence and incidence using a validated administrative data algorithm. Diabetes Care 2002;25:512-16.

22 Vlasschaert MEO, Bejaimal SAD, Hackam DG, et al. Validity of administrative database coding for kidney disease: a systematic review. Am J Kidney Dis 2011:57:29-43.

23 Lopushinsky SR, Covarrubia KA, Rabeneck L, et al. Accuracy of administrative health data for the diagnosis of upper gastrointestinal diseases. Surg Endosc 2007;21:1733-7.

24 Gershon AS, Wang C, Guan J, et al. Identifying individuals with physician diagnosed COPD in health administrative databases. COPD 2009:6:388-94.

25 Gershon AS, Wang C, Guan J, et al. Identifying patients with physician-diagnosed asthma in health administrative databases. Can Respir J 2009;16:183-8.

26 Austin PC, Grootendorst P, Anderson GM. A comparison of the ability of different propensity score models to balance measured variables between treated and untreated subjects: a Monte Carlo study. Stat Med 2007;26:734-53.

27 Brassard P, Lowe A-M, Bernatsky S, et al. Rheumatoid arthritis, its treatments, and the risk of tuberculosis in Quebec, Canada. Arthritis Rheum 2009;61:300-4.

28 Dirac MA, Horan KL, Doody DR, et al. Environment or host?: a case-control study of risk factors for Mycobacterium avium complex lung disease. Am J Respir Crit Care Med 2012;186:684-91.

29 Doucette K, Fishman JA. Nontuberculous mycobacterial infection in hematopoietic stem cell and solid organ transplant recipients. Clin Infect Dis 2004;38:1428-39.

30 Clay H, Volkman HE, Ramakrishnan L. Tumor necrosis factor signaling mediates resistance to mycobacteria by inhibiting bacterial growth and macrophage death. Immunity 2008;29:283-94.

31 Kleinert S, Tony HP, Krueger K, et al. Screening for latent tuberculosis infection: performance of tuberculin skin test and interferon-release assays under real-life conditions. Ann Rheum Dis 2012;71:1791-5.

32 Carmona L, Gomez-Reino JJ, Rodriguez-Valverde V, et al. Effectiveness of recommendations to prevent reactivation of latent tuberculosis infection in patients treated with tumor necrosis factor antagonists. Arthritis Rheum 2005;52:1766-72.

33 Mehta M, Marras TK. Impaired health-related quality of life in pulmonary nontuberculous mycobacterial disease. Respir Med 2011;105:1718-25.

34 Greenberg JD, Reed G, Kremer JM, et al. Association of methotrexate and tumour necrosis factor antagonists with risk of infectious outcomes including opportunistic infections in the CORRONA registry. Ann Rheum Dis 2010;69:380-6.

35 Bykerk VP, Akhavan P, Hazlewood GS, et al. Canadian Rheumatology Association recommendations for pharmacological management of rheumatoid arthritis with traditional and biologic disease-modifying antirheumatic drugs. J Rheumatol 2012;39:1559-82. 